\title{
An error correction framework for sequences resulting from known state-transition models in Non-Intrusive Load Monitoring
}

\author{
Suman Giri, Mario Bergés* \\ Department of Civil and Environmental Engineering \\ Carnegie Mellon University \\ 5000 Forbes Avenue, Pittsburgh, PA, USA
}

\begin{abstract}
Non-Intrusive Load Monitoring (NILM), the set of techniques used for disaggregating total electricity consumption in a building into its constituent electrical loads, has recently received renewed interest in the research community, partly due to the roll-out of smart metering technology worldwide. Event-based NILM approaches (i.e., those that are based on first segmenting the power time-series and associating each segment with the operation of electrical appliances) are a commonly implemented solution but are prone to the propagation of errors through the data processing pipeline. Thus, during energy estimation (the final step in the process), many corrections need to be made to account for errors incurred during segmentation, feature extraction and classification (the other steps typically present in event-based approaches). A robust framework for energy estimation should use the labels from classification to (1) model the different state transitions that can occur in an appliance; (2) account for any misclassifications by correcting event labels that violate the extracted model; and (3) accurately estimate the energy consumed by that appliance over a period of time. In this paper, we address the second problem by proposing an error-correcting algorithm which looks at sequences generated by Finite State Machines (FSMs) and corrects for errors in the sequence; errors are defined as state transitions that violate the said FSM. We evaluate our framework on simulated data and find that it improves energy estimation errors. We further test it on data from 43 appliances collected from 19 houses and find that the framework significantly improves errors in energy estimates when compared to the case with no correction in 19 appliances, leaves 17 appliances unchanged, and has a slightly negative impact on 6 appliances.
\end{abstract}

\footnotetext{
${ }^{*}$ Corresponding author

Email addresses: sumangiri76@gmail.com (Suman Giri), marioberges@cmu.edu (Mario Bergés)
} 
Keywords: NILM, Non-Intrusive Load Monitoring, Energy Management, Energy Efficiency, Energy Estimation

\section{Introduction}

\subsection{Non-Intrusive Load Monitoring and Energy Estimation}

Non-Intrusive Load Monitoring (NILM) is a set of techniques for algorithmically inferring appliance-level energy consumption information using single point sensing of the aggregate energy consumption, typically done at the main electrical panel in a building. Interested readers are encouraged to read 11 and 2] for an overview on the different approaches that have been proposed to tackle the NILM problem. In general, though, there are two main types of approaches that have been suggested in the literature: those that rely on segmenting the aggregate signal according to steady-state changes (event-based approaches), and those that directly model the aggregate signal as a superposition of many source signals corresponding to the appliances (eventless approaches). Though this paper focuses solely on the event-based approaches, it is worth pointing out that there is a growing body of literature on eventless approaches, where latent variable models, such as Factorial Hidden Markov Models (FHMM), are typically employed [3, 4. Hybrid approaches that incorporate elements of both have also been developed (e.g., [5]). Online proceedings of the International and European Workshops on Non-Intrusive Load Monitoring offer a more up-to-date discussion on the latest developments in NILM 6, 7,

The event-based 1 approach to NILM typically involves the following stages: event detection, feature extraction, classification, and energy estimation. Example algorithms that fall under this category, including some by the authors, are $[8,9,10,11,12$. As stated earlier, the overall goal of NILM is to decompose aggregate measurements of power in a building into the power consumed by each of the appliances that are operating during the monitoring period. Given this, after detecting events, extracting their features and classifying them, the final step (energy estimation) produces estimates of the per-appliance power usage, thereby decomposing the aggregate measurements of power for the building.

Briefly, the process runs as follows: first, a signal $S[t]$ (typically active power) is monitored at the aggregate level. Then an event detection algorithm (ED) searches for change-points in $S[t]$, which are assumed to indicate when appliances change their state of operation (this also assumes that each operational state has a relatively stable power consumption during steady state). Following this, a feature-extraction algorithm (FE) extracts features associated with these events. A model is created in advance - through a training phase - to learn a function $(\phi)$ that maps features $(X)$ of the events to labels $(Y)$ corresponding to the appliances (and, optionally, the state transitions) that were responsible for

\footnotetext{
${ }^{1}$ events are significant changes in the aggregate data being monitored (usually active power) that correspond to an appliance changing its state of operation
} 
Energy estimation in supervised NILM is the final step that utilizes the event labels learnt from the classification step and estimates the energy consumed by appliances with the corresponding labels. For example, let $T^{\prime}=\left\{t_{1}^{\prime}, t_{2}^{\prime}, \ldots t_{k}^{\prime}\right\}$ represent the set of event time-stamps in $S[t]$, that results as output from the ED algorithm (here, $k \in \mathbb{N}$ ). Let $T=\left\{t_{1}, t_{2}, \ldots, t_{m}\right\}$, such that $T \subset T^{\prime}$ and $m \in \mathbb{N}$, represent the set of events classified as belonging to appliance $y_{i} \in Y$ after the FE step and subsequent classification using the function $\phi$. Energy estimation for $y_{i}$, then, involves learning the changes in active power that occur during each of the time-stamps in $T$. These are also referred to as power deltas, and are typically extracted by calculating the difference in aggregate active power (denoted by $\Delta P_{j}$ ) over a certain window around the event time-stamp $\left(t_{j} \in T\right)$. Once all $\Delta P_{j}$ values for $y_{i}$ are calculated, an ordered sequence of power deltas results. Using this, the power trace for $y_{i}$ can be constructed by assuming piece-wise constant power between each element of this sequence, along with an initial power value $P_{0}$, and then performing the following sum to obtain the power value at time-stamp $t$ :

$$
\hat{P}[t]=P_{0}+\sum_{j=1}^{|R|} \Delta P_{j}
$$

where $|R|$ denotes the cardinality of set $R=\left\{t_{j}: t_{j}<t ; \forall t_{j} \in T\right\}$. Energy estimation for $y_{i}$ is then simply the sum of the power trace over the time of interest ( say $t_{1}$ to $t_{m}$ ), i.e.,

$$
E_{i}=\sum_{t_{1}}^{t_{m}} \hat{P}[t]
$$

The energy estimates can, however, be affected by errors resulting from different algorithmic steps in NILM. In [13, the authors presented a method to build Finite State Machine (FSM) models for appliances based on their power delta sequence. The FSM model is a directed graphical representation with the nodes representing the different states that can occur within an appliance and the edges representing the possible transitions between them. Figure 1 . taken from [13] shows the FSM models for a Fridge, TV, and Laptop from a publicly available dataset called BLUED [14. Using the FSM model, it is possible to constrain the power trace and energy estimates resulting from power delta sequences to mitigate some of the errors resulting from NILM. A robust framework for energy estimation should use the labels from classification to (1) model the different state transitions that can occur in an appliance; (2) account for any misclassifications by correcting event labels that violate the extracted 

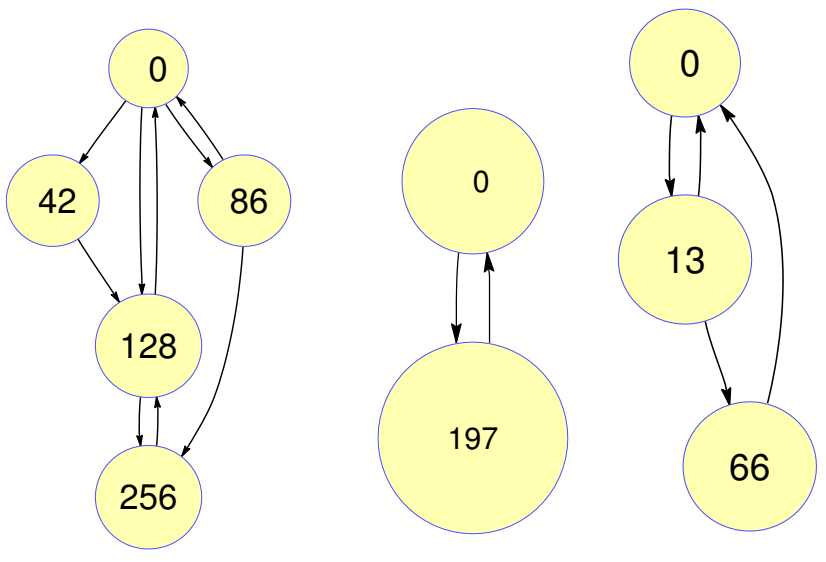

Figure 1: FSM diagram for a Refrigerator from BLUED-1 (left), TV from BLUED-1 (center) and Laptop from BLUED-2 (right). The numbers denote the power consumption (as measured in Watts) in the different states of operation that the appliances can be in, and the arrows denote possible transitions between these states.

model; and (3) accurately estimate the energy consumed by that appliance over a period of time. In this paper, we extend an error-correction step in the framework presented in 13 by introducing an improved algorithm which looks at sequences generated by Finite State Machines (FSMs) and corrects for errors in the sequence. Here, errors are defined as state transitions that violate the FSM.

\subsection{The need for error correction}

The output of the classification step in supervised NILM is a time series of state-transition labels assigned to the observed events. It is trivial to extract ordered sequences of such labels pertaining to each appliance from this time series. Each such sequence will be denoted as $C_{\text {seq }}$. We assume that an operating model for the appliance that represents each such sequence is also available in the form of an FSM. Typically, this means that information about possible states, and state transitions for the appliance is available. Authors in [13] present a method to extract such information from data. Figure 2 presents a graphical summary of the steps involved in the framework, and how error correction fits in the bigger picture. The sequence $C_{s e q}$ is a direct product of the event detection and classification steps and errors occurring in these steps (e.g., missed events, misclassifications, etc.). This results in a sequence that violates the FSM that generates it, and causes erroneous energy estimation results. Hence, an additional step of error identification and correction is required before energy estimation is performed.

The problem of correcting errors in sequences generated by FSMs has been studied extensively for problems in communication theory, DNA sequencing, 


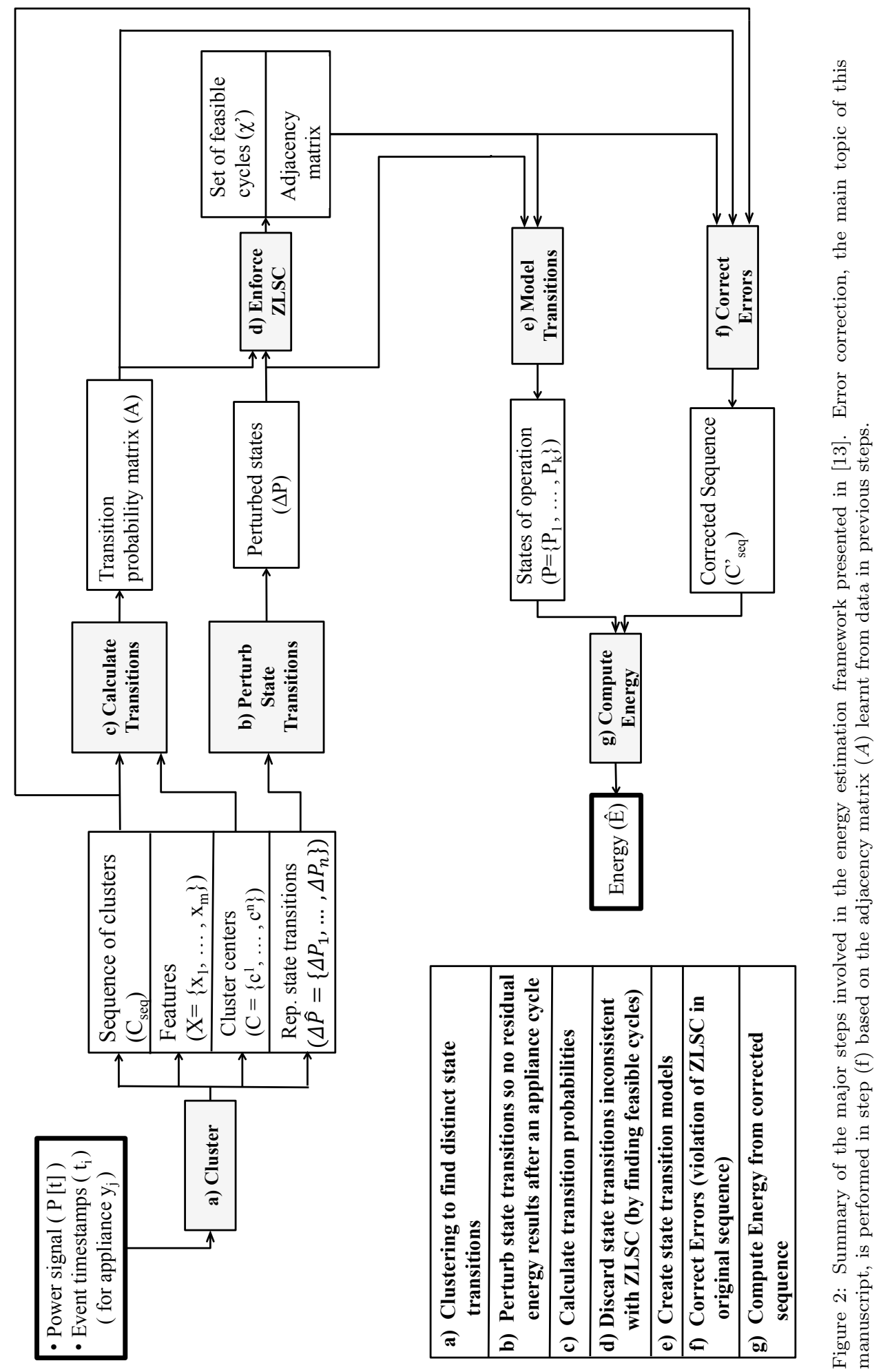


pattern recognition, etc 15, 16. Hart drew similarities between the NILM problem and the problem of decoding additive signals on a Multiple-Access Channel (MAC) 17. He cited the low signal to noise ratio, and low message rate to channel capacity ratio as some of the desirable features of this channel. Recently, authors in [18] have begun to treat the event-based disaggregation problem from the perspective of the nascent field of Graph Signal Processing (GSP) [19, 20, 21]. In this context, error correction is performed by adding a regularizer to the problem of finding the optimal graph for total graph variation minimization. The authors report that a total graph variation regularizer (i.e., one that smooths the event label sequence) alone produces solutions that are too smooth, and incorporated simulated annealing as an error-correction proxy to refine the results. We see many connections between the work presented here and GSP-based approaches to the problem, but leave it as future work to investigate these links in more detail.

Compared to other decoding problems where error correction is required, the NILM channel presents a unique set of challenges. Typically, solutions for correcting errors assume certain properties about the noise in the channel, which makes the correction process more systematic. In addition, most error correcting algorithms have a coder for the input sequence that allows it to be encoded in a specific way to facilitate decoding. The NILM problem does not allow for either of these steps, as it has no control over the input sequence. Essentially, the only flexibility that exists is the capacity to exploit information about the constraints imparted by the FSM to correct for errors. It is also worth noting that the definition of the channel in this problem not only includes the NILM hardware, but also the process by which the algorithm arrives at appliance specific time-series. Finally, since the goal of correcting errors in NILM is to perform energy estimation, errors are highly sensitive to propagation, and hence the error-correction algorithm for such errors needs to minimize such possibilities.

To get a better understanding of this, assume an appliance with three states of operation labelled as cluster assignments 1,2 , and 3 corresponding to $\Delta P$ values of $40,-40$ and -20 respectively. Say, the transition probability matrix is given by $\left[\begin{array}{ccc}0 & .5 & 0 \\ .1 & 0 & .6 \\ 1 & 0 & 0\end{array}\right]$. Also, assume a sequence of events occur as $C_{\text {seq }}=\left(\begin{array}{l}1 \\ 1\end{array} 121212\right)$. Now, the net power consumption of the appliance at each of the events are (400400400). For simplicity, if we assume the units of the power deltas to be in kilowatt-hour $(\mathrm{kWh})$, and if each event lasts for 1 hour, the total energy consumption for the appliance, over the span of 6 hours, is $120 \mathrm{kWh}$. Now, assume there was an error in $C_{\text {seq }}$ resulting from misclassification, and the third event was classified as a 2 instead of 1 , resulting in $C_{\text {seq }}=(122212)$. This means the net power consumption would now be $(40 \quad 0-40-80-40-80)$, and the energy consumption under previous assumptions would be $40 \mathrm{kWh}$. 2

\footnotetext{
${ }^{2}$ negative energy consumption were treated as zeros.
} 
As can be seen, due of one error in the sequence, the energy estimates were off by 3 times. An ideal error correction algorithm should be able to recognize this and replace the erroneous state with its proper substitute.

Hart also proposed an algorithm to correct errors in sequences generated by FSMs in 22 where he mentioned NILM as one of the potential use cases for his algorithm. His framework introduces the idea of channel rules, where the user knows, a priori, the kinds of modifications the channel-inverter can impart on the observed sequence. We modify his concept to allow for the same channel rules (namely single bit insertion, deletion or substitution) for all sequences, which makes the method more generalizable. In addition, we introduce constraints specific to energy estimation in the algorithm, which caters to the ultimate goal of the error correction. In summary, the major contributions of this paper are: (a) an extended algorithm for correcting errors in supervised NILM with novel evaluation metrics, and (b) results from real data from 43 appliances collected from 19 different houses.

\subsection{Introduction to the framework}

Our framework for error correction is based on the concept of minimizing the global cost of correction for errors in a sequence. Ultimately, this reduces to solving the shortest path problem in a directed acyclic graph. Following are the notations that will be used:

For rest of the paper, the notations will refer to events occurring for a particular appliance $y_{i}$. As summarized in Figure 2, once, all the features $(X)$ are extracted from events occurring at time-stamps $T$, they are clustered using affinity propagation algorithm. $C=\left\{c^{1} \ldots c^{n}\right\}$ denotes the set of labels for the $n$ clusters that result (with $n \in \mathbb{N}$ ). Let $C_{\text {seq }}=\left\{c_{1}, c_{2} \ldots c_{m}\right\}$ represent the ordered sequence of cluster assignments for all the events such that $c_{k}$ denotes the cluster label associated with the state transition that occurs at event timestamp $t_{k}$. Let $A$ denote the transition probability matrix learnt from the FSM, where $a_{i j} \in \mathbb{R}$ denotes the probability of transition from state $c^{i}$ to state $c^{j}$. If $c_{k-1}=c^{i} ; c_{k}=c^{j} ; \forall c^{i}, c^{j} \in C$, an error occurs whenever $a_{i j}=0$.

The first step is to model the cost functions. Costs can be of different types based on the kind of error correction being done. For error correction using substitution, where a faulty state is replaced by another state which has a valid transition, we define errors as the negative logarithm of transition probabilities, i.e.

$$
\chi_{i, j}^{S}=\log \left(-\gamma\left(a_{i j}\right)\right)
$$

Here the superscript $S$ denotes the cost for substitution between states $c_{k-1}=$ $c^{i} ; c_{k}=c^{j} ; \forall c^{i}, c^{j} \in C$, and net $\gamma$ is a constant that alters the probability of substitution based corrections. This ensures that transitions with low probabilities will have a higher associated cost when replacing an erroneous term, and vice versa. Transitions that are not possible, will have infinite associated cost. 
Costs for insertion of a new state, and deletion are also calculated.

For insertion, we calculate the joint probability of the new state being inserted, again, $c_{k}$, coming after its preceding state, $c_{k-1}$ and before the succeeding state, $c_{k+1}$. As before, assume $c_{k-1}=c^{h} ; c_{k}=c^{i}$, and $c_{k+1}=c^{j} ; \forall c^{h}, c^{i}, c^{j} \in C$. The probability is $a_{h i} a_{i j}$. Depending on the prior knowledge we have about the process, i.e. belief on the kinds of errors that can occur, the cost function for inserting a state $c^{i}$ before erroneous transition from $c^{h}$ to $c^{j}$ is calculated as

$$
\chi_{i, j}^{I}=\log \left(-\mu\left(a_{h i} a_{i j}\right)\right)
$$

where $\mu$ is a constant (typically a small number) that alters the probability making insertions less likely based on prior belief. The superscript $I$ denotes that the cost is for insertion.

Similarly, for deletion, we calculate the cost using the transition probability from the state before the one being deleted to the state after it. Extra cost is added to make deletions more or less likely depending on prior knowledge. Using the same notation as before, if $c^{k}$ is to be deleted, mathematically, this amounts to calculating,

$$
\chi_{i, j}^{D}=\log \left(-\nu\left(a_{h j}\right)\right)
$$

The variable $\nu$ is again a constant similar to $\mu$, and the superscript $D$ denotes the cost for deletion, with $c^{i}$ being the state that is deleted for correcting the error.

The goal is to correct errors, as defined above, by making changes that minimize the cost of correction. For instance, a local greedy algorithm, as proposed in 13. can result in error propagation. An ideal algorithm will be able to correct for local errors while ensuring that the effect of the correction does not result in propagation of error. In the example presented in Section 1.2 the error occurring at the second event in $C_{\text {seq }}=\left(\begin{array}{l}1 \\ 2\end{array} 2212\right)$, could be solved by checking the transition probability for the most likely state, which would involve replacing the erroneous state (2) in the third event with the state with the highest probability (3), resulting in $C_{\text {seq }}=\left(\begin{array}{l}1 \\ 2\end{array} 32212\right)$. This would, however, introduce another error, as the transition from 3 to 2 is not possible. So, the algorithm would iterate again resulting in $C_{\text {seq }}=(123112)$. After several iterations, the final sequence will converge to $C_{\text {seq }}=(123123)$, with energy consumption of $60 \mathrm{kWh}$. So, even though the resulting sequence does not have "errors", the error correction at a single point has resulted in a propagation of errors which has affected the energy estimate. An ideal error correction algorithm will be able to account for such propagation of errors resulting from correction steps in the sequence, and choose the optimal correction step (which in this case would be to replace 2 with a 1 in the third event in $C_{\text {seq }}$.

To tackle this problem, we represent $C_{\text {seq }}$ as a fully connected network diagram 


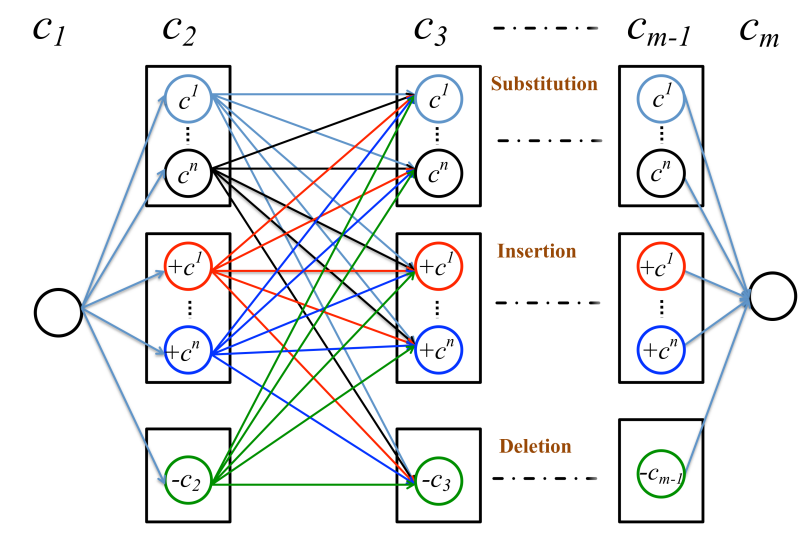

Figure 3: Network flow diagram for the sequence $C_{\text {seq }}=\left\{c_{1} \ldots c_{m}\right\}$. Three different kinds of error corrections are possible: Substitution, Insertion and Deletion.

as shown in Figure 3. For an error point $c_{i}$ in $C_{\text {seq }}$ there are multiple error correcting steps possible, each with specific costs denoted by the matrix $\chi$ of dimension $m \times(2 n+1)$. Throughout this paper, we use $\chi_{i j}$ to denote the cost element in row $i$ and column $j$ of $\chi$. The goal will be to find a feasible path from $c_{1}$ to $c_{m}$ with the least cost possible. We achieve this by modeling the problem as a special case of the min-cost flow problem in Network theory, known as the shortest path problem 23. The basic idea behind the problem is to find the shortest path between two nodes in a directed graph, when the distance between all connected nodes (also known as weights or cost) is known. Several algorithms have been proposed to solve the problem, with Dijkstra's algorithm being perhaps the most well known and simplest in terms of implementation 24. In the next section, we formalize this optimization problem.

\section{Shortest-path formulation}

Notationally, we have the following optimization framework for the shortestpath formulation of the error correcting algorithm. As depicted in Figure 3. $C_{\text {seq }}$ is the erroneous sequence. The source node is $c_{1}$ and the destination node is $c_{m}$. The goal is to find the shortest path from the source to the destination. Say $d_{u v}$ is a variable that denotes whether an edge lies in the shortest path or not. So, $d_{u v}=1$ if the edge between node $u$, denoted by $c_{u}$ and node $v$ denoted by $c_{v}$ lies in the shortest path. The way the network flow is arranged in this particular formulation, $v=u+1$. The source node is denoted by $c_{1}$ and the destination node denoted by $c_{m}$. As mentioned before, $c_{u}, c_{v} \in C$. The shortest 
path algorithm optimizes:

$$
\min \sum_{u, v}\left(d_{u v} \chi_{u v}\right)
$$

subject to: $\quad d_{u v} \geq 0 \forall u, v$
$\sum_{u} d_{u v}-\sum_{v} d_{u v}=\left\{\begin{aligned} 1 & \text { if } u=1 \\ -1 & \text { if } u=m \\ 0 & \text { otherwise }\end{aligned}\right.$

The unimodularity of the constraint co-efficient matrix allows the relaxation of the inherent integer constraints in the setup, while still ensuring that all solutions are either 0 or 1 . Proof and further details can be found in [25]. There are several algorithmic approaches to solving this optimization problem. We use, what is perhaps, the most popular option, known as the Dijkstra's algorithm. The computational complexity of Dijkstra's algorithm is $\mathcal{O}\left(V^{2}\right)$, where $V$ is the total number of vertices in the graph. For sequence $C_{s e q}$, $V=m(2 n+1)$, and hence the computational complexity in using Dijkstra's algorithm is $(m(2 n+1))^{2}$, where $n=|C|$ is the total number of possible states. The number of vertices can be pruned using some prior knowledge about state transitions, and by removing certain nodes in the sequence that are clearly not possible (for instance, edges with $\chi_{u v}=\infty$ ). Worth noting is that there are variations of Dijsktra's methods (d-way heap, Fibonacci heap, etc.), that can solve the shortest path problem faster, depending on the setup of the network and the kinds of data structures used [25, 26]. Since computational complexity of the algorithm is not a major limiting factor for the length of sequences typically encountered in NILM, we only explore the standard version of the algorithm.

The shortest path calculated using Dijkstra's algorithm incorporates information about state transitions, but does not yet utilize the constraints resulting from the possible states in the FSM. So, for instance, the net energy consumption at any given point for an appliance can still be more than the maximum allowed state for that appliance. To incorporate this information, we employ an iterative algorithm that changes the cost function at specific points in the shortest path. The idea, as depicted in Algorithm 1 is to iteratively identify nodes that allow the aggregate net power consumption to be in violation of the maximum or minimum possible state, and locally set the cost for the immediate edge that leads to such node to infinity. The input to the algorithm is the sequence $C_{\text {seq }}$, the FSM states (obtained after step (e) of the framework in Figure 2), and the adjacency matrix (resulting from enforcing the ZLSC constraint in step (d) of the framework). Then the shortest path is recalculated, and the violation of states is checked for again. The algorithm terminates after a shortest path with no violation is found. 


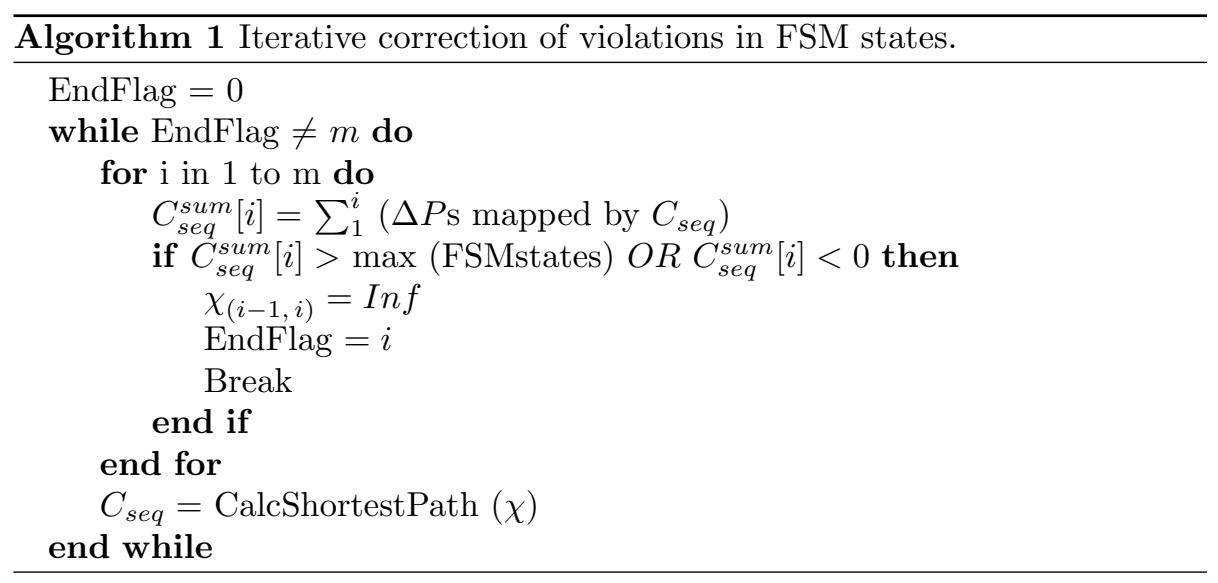

\section{Evaluation}

In this section, we evaluate the effect of using this error correcting formulation on $C_{s e q}$-like sequences originating in supervised NILM. Given that the ultimate goal of the corrected sequence is to perform energy estimation (i.e. calculate $E_{i}$ for appliance $y_{i}$ ), we evaluate the capacity of the framework to both recreate a sequence that is as close as possible to the original sequence, and to estimate energy as closely as possible.

\subsection{Evaluation metrics}

We denote the erroneous sequence as $\left.C_{s e q}^{E}\right]^{3}$, the corrected sequence as $C_{s e q}^{C}$ and the uncorrupted (original) sequence as $C_{s e q}^{C}$. To evaluate how well the error correction framework works, we need some metric to compute the similarity between $C_{\text {seq }}^{C}$ and $C_{\text {seq }}^{O}$. Traditional metrics to evaluate the proximity of time series sequences like Euclidean distance, or RMS error fail in this case because insertions and deletions may lead to $C_{s e q}^{C}$ and $C_{s e q}^{O}$ which have different lengths. To get around this limitation, we leverage the Dynamic Time Warping (DTW) distance measure, which is basically a metric to compute the similarity between two temporal sequences of different lengths. DTW has been used to compute the similarity of sequences that might be warped non-linearly in time in applications ranging from shape matching to speaker recognition [27, 28]. Authors in 29] have also proposed an unsupervised solution to NILM based on DTW, where it is used for template matching.

Say $X=\left(x_{1}, \ldots, x_{A}\right)$ and $Y=\left(y_{1}, \ldots, y_{B}\right)$ are two time-series sequences of length $A$ and $B$ respectively where $A, B \in \mathbb{N}$. Further, assume that the two sequences are from some vector space $V$ such that $x, y \in V$. A cost metric is then defined as a function $f$ that performs the following mapping: $f: V \times V \rightarrow \mathbb{R}^{+}$.

\footnotetext{
${ }^{3}$ so far, we have denoted it as simply $C_{\text {seq }}$
} 


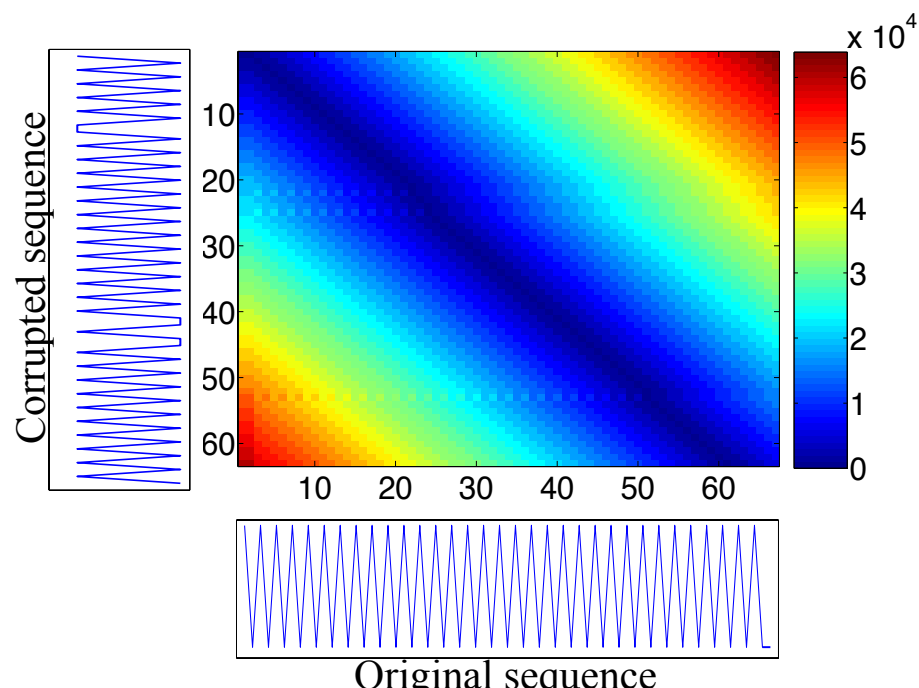

Figure 4: DTW cost matrix for two sequences (original and corrupted) for a washer (label: 3 ) in dataset REDD-1. The appliance only has two states, and hence the sequence fluctuates between two values.

Upon calculating the cost metric for all possible pairs of items in sequences $X$ and $Y$, a cost matrix $F \in \mathbb{R}^{\mathbb{A} \times \mathbb{B}}$ results. The DTW algorithm attempts to find an alignment between sequences $X$ and $Y$ that minimizes the overall cost. The optimal cost of alignment becomes the DTW distance. For the obvious case where $X$ and $Y$ are the same sequence, the DTW distance will be zero. Figure 4 shows an example of two time series sequences with corresponding cost matrix, and Figure 5 shows the shortest path of alignment in the cost matrix.

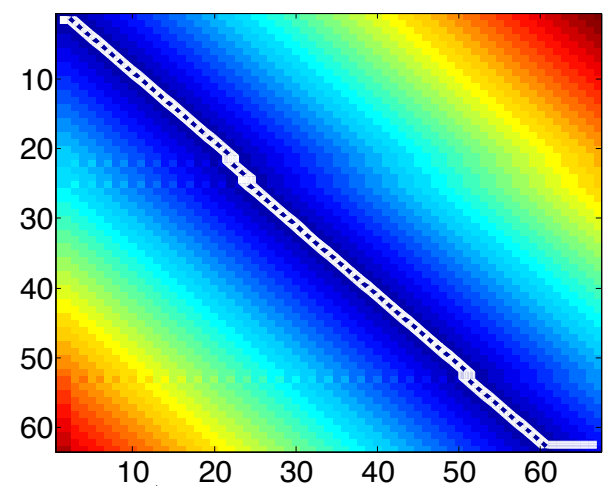

Figure 5: Path (shown in white) with minimal cost of alignment for the original sequence and corrupted sequence as shown in Figure 4. The DTW distance was 5808.

In addition, the error correction framework imparts certain appliance specific constraints to $C_{s e q}^{E}$ to facilitate energy estimation. To evaluate the efficacy of those constraints we compute the energy consumed by the appliance as given 
by $C_{\text {seq }}^{C}$ (denoted by $\hat{E}$ ) and compare it with the actual energy consumed by the appliance (denoted by $E$ ). The evaluation metric here is simply a percentage error as given by

$$
\mathbf{e}=\frac{E-\hat{E}}{E} \times 100 \%
$$

\subsection{Evaluation on Simulated Data}

We evaluated the framework on both real and simulated data based on the metrics discussed. For simulation, we created an appliance model which, in turn, generated the sequence of state transitions while preserving appliance like properties (e.g., ZLSC ${ }^{4}$ ). The idea behind the simulations was to study how different parameter values for cost constants affect the corrected sequence. In addition, for testing on real data, we evaluated our model on 43 different appliances collected from 19 different houses.

\subsubsection{Appliance model}

The following are the major components of the simulated appliance model; these are also summarized in Table 1:

- Number of states $\left(n_{1}\right)$ : A random number of states of operation (between 2 and 9$). n_{1} \sim \mathcal{U}\{2,9\}$

- Power consumption $\left(P_{i}\right)$ : Random values between 50 and 3000 assigned to each state. $P_{i} \sim \mathcal{U}(50,3000)$

- Transition probability $\left(A_{1}\right)$ : A doubly stochastic hollow matrix of dimension $n_{1} \times n_{1}$ with random values between 0 and 1 .

- Sequence length $(m)$ : A variable set to 500 to represent the number of events registered for the appliance. This is typically adequate to capture appliance behavior.

- State Sequence $\left(C_{s e q}^{s t}\right):$ A sequence of dimension $m \times 1$ generated using hmmgenerate function in MATLAB given $A_{1}$.

- Original State transition sequence $\left(C_{s e q}^{O}\right): C_{\text {seq }}^{O}=Z \times C_{\text {seq }}^{\text {st }}$, where $Z$ is an $m \times m$ matrix (sometimes also called a difference matrix) of the following form:

$$
\left(\begin{array}{ccccccc}
1 & -1 & 0 & 0 & 0 & \ldots & 0 \\
0 & 1 & -1 & 0 & 0 & \ldots & 0 \\
0 & 0 & 1 & -1 & 0 & \ldots & 0 \\
\vdots & \vdots & \vdots & \vdots & \vdots & \vdots & \vdots \\
0 & 0 & 0 & 0 & \ldots & 1 & -1
\end{array}\right)
$$

${ }^{4}$ ZLSC: The Zero Loop Sum Constraint, first coined by Hart, stipulates that the sum of all possible power transitions in a cycle should equal zero. A cycle occurs when an appliance in one state, undergoes some state transitions, and returns to the same state. 
- Number of state transitions $(n)$ : Number of unique values in the state transitions sequence.

- Transition probability $(A)$ : A matrix of dimension $n \times n$, calculated using the the frequency of transition from each state to another in $C_{s e q}^{O}$. This can also be computed using hmmestimate in MATLAB.

- Emission probability $(B)$ : A randomly generated doubly stochastic matrix of dimension $(n \times n)$ with diagonal elements set to random values close to 1.The rest of the matrix has random values less than 0.1 . This ensures that most of observed sequence come out as the original sequence with potential for a few errors.

- Observed State Transition Sequences $\left(C_{\text {seq }}^{E}\right)$ : A sequence with elements sampled from the probability distribution of corresponding elements in $C_{\text {seq }}^{O}$ given $B$. When state 1 (out of $n$ states) is observed in $C_{\text {seq }}^{O}$ but not in its corresponding position in $C_{\text {seq }}^{E}$, it is an insertion error. Alternatively, if state 1 appears in $C_{\text {seq }}^{O}$ but not in its corresponding position in $C_{\text {seq }}^{O}$, it is a deletion error. All state 1 values are subsequently removed from both sequences.

- Duration $(\tau)$ : A sequence of length $m$ with values between 1 and 7200 seconds, assigned to each state transition. $\tau \sim\{1,7200\}$.

\subsubsection{Results}

We ran the simulation on different combinations of Insertion, Deletion and Substitution cost constants $(\mu, \nu$, and $\gamma)$. For each combination of cost constants (ranging from 1 to 50 each), we performed 100 simulations each, resulting in a total of $1.25 \times 10^{7}$ trials. The overall goal was to compare post error correcting framework sequence to a case without error correction, according to the metrics defined previously- namely DTW distance, RMS error, and final energy estimation results. Figures 6 and 7 show a color map of the effect of different $\mu$ and $\nu$ parameters on the energy estimation accuracy results for a specific instance of $\gamma(\gamma=16)$. The figure indicates that the energy estimation error improves consistently after the deletion cost crosses a certain a threshold (roughly 15). This is most likely the point at which deletion corrections become costlier than substitution corrections, and hence more substitutions are made where possible. Given substitution errors are more likely to exist in the simulation, this improvement makes sense. Higher cost values, however, seem to be produce more unstable results as depicted by the standard deviation color map in Figure 7. Again, this makes intuitive sense, as once the cost values for a certain kind of correction cross a certain threshold, that type of correction is avoided, which results in a certain kind of error not being accounted for throughout the sequence.

Figure 8 shows how the three metrics vary before and after the framework is used for a specific instance of the three costs values $(\mu=16, \nu=16, \gamma=12)$. 
Table 1: Summary of the parameters used in the simulation model

\begin{tabular}{|c|c|c|c|}
\hline Parameter & Value & Parameter & Value \\
\hline \# of states & $\boldsymbol{n}_{\mathbf{1}} \sim \mathcal{U}\{2,9\}$ & $\begin{array}{l}\text { Power } \\
\text { consumption }\end{array}$ & $\boldsymbol{P}_{\boldsymbol{i}} \sim \mathcal{U}(50,3000)$ \\
\hline $\begin{array}{l}\text { Transition } \\
\text { probability }\end{array}$ & $\begin{array}{l}\boldsymbol{A}_{\mathbf{1}} \sim \text { random } \\
\text { doubly stochastic } \\
\text { hollow matrix of } \\
\text { size } n_{1}\end{array}$ & $\begin{array}{l}\text { Sequence } \\
\text { length }\end{array}$ & $\boldsymbol{m}=500$ \\
\hline $\begin{array}{l}\text { State } \\
\text { Sequence }\end{array}$ & $\begin{array}{l}C_{\text {seq }}^{\text {st }} \sim \\
\text { hmmgenerate } \\
\text { given } A_{1}\end{array}$ & $\begin{array}{l}\text { Original } \\
\text { state } \\
\text { transition } \\
\text { sequence }\end{array}$ & $C_{\text {seq }}^{O}=Z \times C_{s e q}^{s t}$ \\
\hline $\begin{array}{l}\text { \# of state } \\
\text { transitions }\end{array}$ & $\begin{array}{l}\boldsymbol{n}=\# \text { of unique } \\
\text { values in } C_{\text {seq }}^{O}\end{array}$ & $\begin{array}{l}\text { Transition } \\
\text { probability }\end{array}$ & $\begin{array}{l}\boldsymbol{A} \sim n \times n \text { matrix } \\
\text { computed using } \\
C_{\text {seq }}^{O}\end{array}$ \\
\hline $\begin{array}{l}\text { Emission } \\
\text { probability }\end{array}$ & $\begin{array}{l}\boldsymbol{B} \sim \text { Randomly } \\
\text { generated doubly } \\
\text { stochastic matrix } \\
\text { of size } n\end{array}$ & $\begin{array}{l}\text { Observed } \\
\text { state } \\
\text { transition } \\
\text { sequence }\end{array}$ & $\begin{array}{l}C_{\text {seq }}^{\boldsymbol{E}} \sim \text { Sequence } \\
\text { sampled using } B \\
\text { and } C_{\text {seq }}^{E}\end{array}$ \\
\hline Duration & $\boldsymbol{\tau} \sim\{1,7200\}$ & & \\
\hline
\end{tabular}

The lines in the middle of the box plots in Figure 8 indicate the median values of the logarithmic of the metrics of interest after 1000 trials each, with the box-margins denoting the limiting values for $75^{t h}$ and $25^{t h}$ percentile each. The whiskers represent the minimum and maximum values in each of the simulation. Although, no statistically significant change is observed as the number of states are varied, it is obvious that using the error error correction improves results significantly as measured by all three metrics. The fact that the DTW metric is consistently less after the framework is applied means that the corrected sequence is closer to the original sequence than the corrupted sequence. In addition, we compared the power traces of the power signals created using the corrupted sequence and the corrected sequence, and computed the RMS error. As expected, the corrected sequence had much lower RMS error values. All energy estimation errors were found to be less than $40 \%$ for corrected sequences, which highlights the utility of the method. Although these plots show the effect of the framework for one combination of the costs, the results from Figure 6 confirms that a wide range of parameter selection would still result in better energy estimates. The major takeaway from the simulation results is that error correction results in corrected sequences that are much closer to the original sequence than the corrupted sequence, and in much better energy estimates. Moreover, this performance is not limited to a significant value of the cost parameters, but is tolerant over a wide range. 


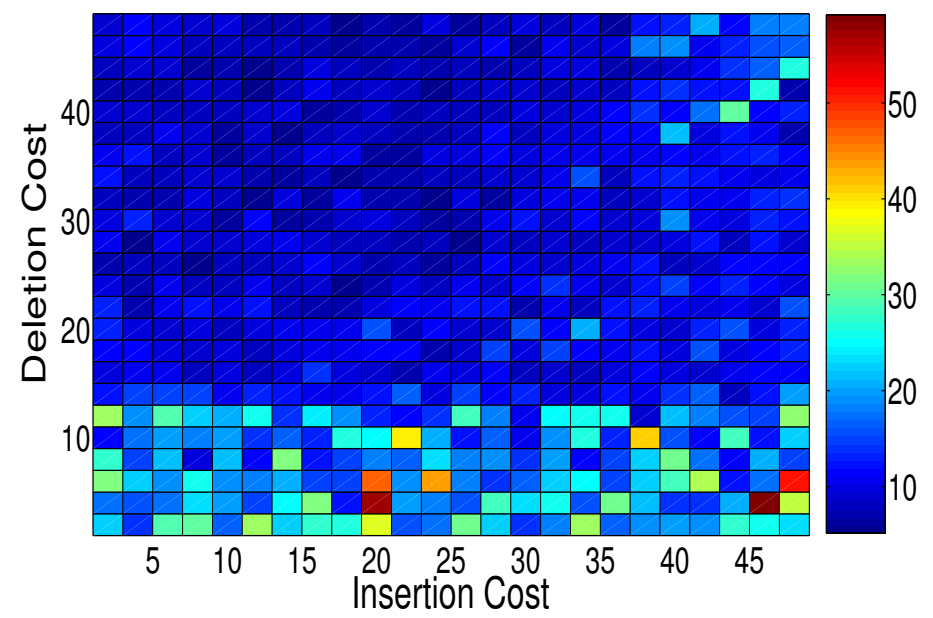

Figure 6: Median error in energy estimation (e) for varying values of $\mu$ and $\nu$, with $\gamma=16$. The medians were calculated after 100 simulations of each cost combination.

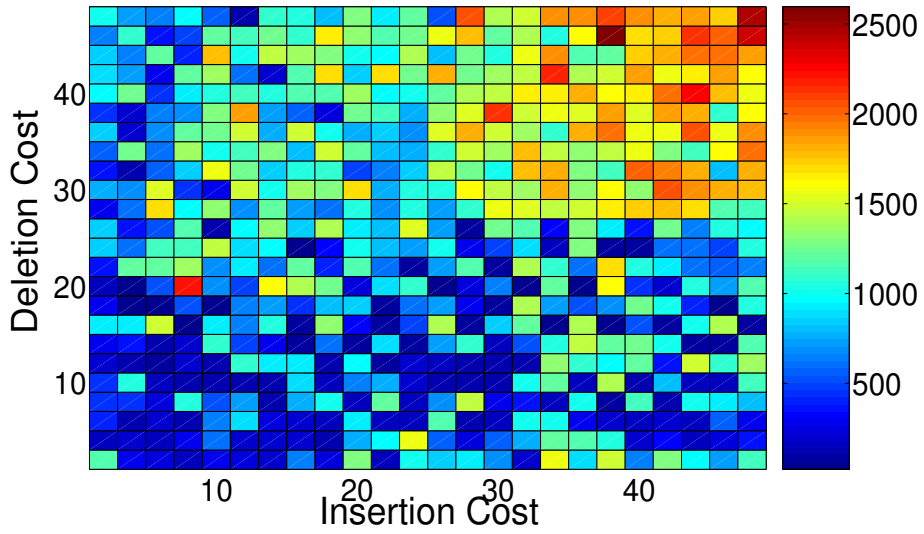

Figure 7: Standard Deviations for the median errors as reported in Figure 6 for 100 iterations of different cost combinations.

\subsection{Evaluation on real data}

We evaluated the error correction framework on 43 appliances from 2 different datasets comprising of 19 houses. To our knowledge, only one publicly available dataset (BLUED-1) has event-level ground truth data 14]. We have added two more datasets to the BLUED series, namely BLUED-2 and BLUED3 which was collected by authors in [30. All three datasets are collected in the same format $(60 \mathrm{~Hz}$ real and reactive power). In addition, we took another publicly available dataset- REDD- and labelled all the events for appliances of interest in House 1 [31. This dataset was collected at a frequency of $1 \mathrm{~Hz}$ real power. Since appliance-level ground truth was not available in this dataset, we had to label events at sub-circuit level. As a consequence, only appliances that 


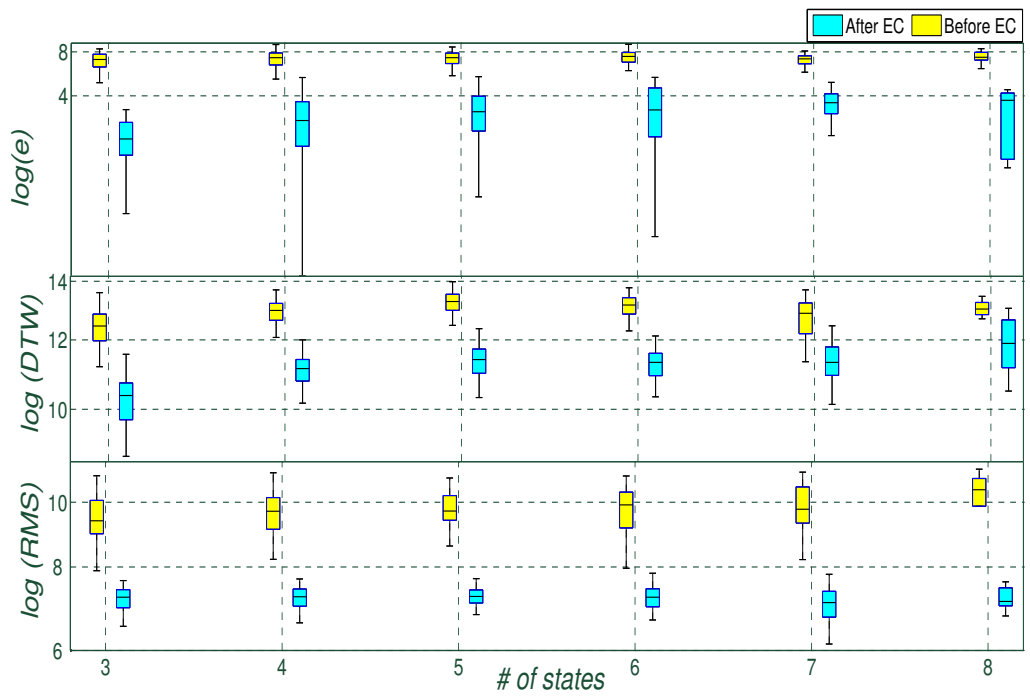

Figure 8: Box plots of logarithmic values of the energy estimation error: e (top), DTW distance of corrected sequences (middle), and RMS error of reconstructed power traces (bottom), with box margins representing the 25 th and 75 th percentile values, and median was calculated after 1000 simulation trials. EC stands for Error Correction.

The framework described in 13 was used to generate the sequence of state transitions $C_{s e q}$ from the sequence of labelled events available for these appliances. This process also has the potential to introduce additional errors in the sequence. This could be because of miscalculation of state transition values due to simultaneous events, or other factors like erroneous clustering, etc. Since no "ground truth" was available for $C_{s e q}$, we only evaluated the framework for its ability to estimate energy, and compared it to the case when no error correction was done. The same methods described in 13 were used to compute energy values from resulting sequences. The error metric described in Equation 7 was used to compute error (e) after correction. In addition, we also computed the error in energy estimation without the error correction algorithm (denoted by $e^{\prime}$ ) using energy estimates done through the erroneous sequence $C_{\text {seq }}^{E}$. The 


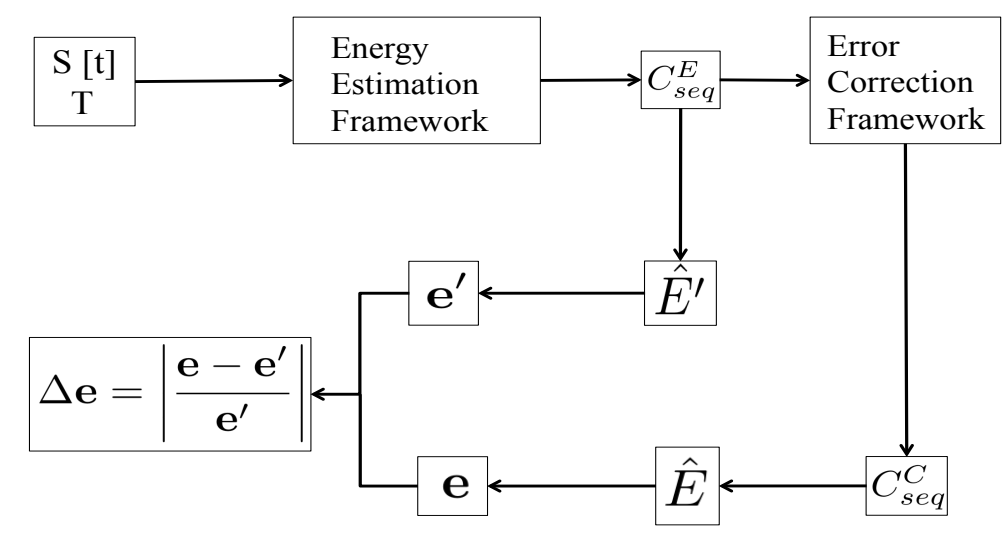

Figure 9: The inputs and outputs involved in the evaluation process when the error correction is applied on BLUED and REDD datasets. Energy estimation framework refers to the framework from [13, and Error correction framework is the one proposed in this paper.

energy estimate computed using $C_{\text {seq }}^{E}$ is denoted by $\hat{E}^{\prime}$.

$$
\mathbf{e}^{\prime}=\frac{E-\hat{E}^{\prime}}{E} \times 100 \%
$$

To understand the effect of using the error correction framework, and its impact on the energy estimation errors, we created a metric which we call the improvement ratio (denoted by $\Delta \mathbf{e}$ ), defined as follows:

$$
\Delta \mathbf{e}=\left|\frac{\mathbf{e}-\mathbf{e}^{\prime}}{\mathbf{e}^{\prime}}\right|
$$

The improvement ratio denotes the fraction of the erroneous energy estimates that is corrected for by the use of the error correction algorithm. Values close to 1 are good because they indicate the error correction algorithm accounted for most of the errors, and values above 1 denote cases where the error correction algorithm worsened the energy estimates. Values of zero denote cases where the error correction algorithm does not affect the energy estimates. It is also to be noted that $e^{\prime}$ will rarely equal zero, as it is extremely unlikely that the energy estimates are precisely the same as the ground truth. Figure 9 summarizes the inputs and output of this evaluation process.

\subsubsection{Results}

The error correction framework was evaluated on the aforementioned datasets. Table 3 depicts the actual values after the framework was applied on 43 appliances. Again, the framework for energy estimation was the same as the one used by the authors in [13. As summarized in Figure 9, the energy estimation framework first generated $C_{s e q}$ values for each appliance from the list of labeled events that it was provided. The estimated energy consumption of those appliances were first computed using $C_{s e q}$ only, and then compared to the case when 
Table 2: Summary of the datasets used for evaluation in the paper. Dataset index B stands for BLUED and R stands for REDD. Appliances are indexed as follows: A-Refrigerator; B-Lights; C-TV; D-Computer; E-Laptop; F-AV System; G-Washer; H-Oven; I-Dishwasher; J-Microwave; K-Dryer; L-Furnace; M-PoolPump; N-HairDryer. Column 5 lists the number of events (state-transitions) associated with the corresponding appliance.

\begin{tabular}{|c|c|c|c|c|}
\hline $\begin{array}{l}\text { Data- } \\
\text { set }\end{array}$ & $\begin{array}{c}\text { Sampling } \\
\text { Freq. } \\
(\mathrm{Hz})\end{array}$ & $\begin{array}{l}\text { Duration } \\
\text { (days) }\end{array}$ & Appliances used & \# of events \\
\hline B-1 & 60 & 7 & {$[\mathrm{~A} ; \mathrm{B} ; \mathrm{C} ; \mathrm{D} ; \mathrm{E} ; \mathrm{F}]$} & {$[616 ; 26 ; 54 ; 45 ; 14 ; 8]$} \\
\hline B-2 & 60 & 7 & {$[\mathrm{~A} ; \mathrm{E} ; \mathrm{C} ; \mathrm{G}]$} & {$[287 ; 53 ; 289 ; 1897]$} \\
\hline B-3 & 60 & 7 & {$[\mathrm{~A} ; \mathrm{E} ; \mathrm{C} ; \mathrm{G}]$} & {$[287 ; 53 ; 289 ; 1897]$} \\
\hline $\mathrm{R}-1$ & 1 & 26 & {$[\mathrm{~A} ; \mathrm{E} ; \mathrm{C} ; \mathrm{B} ; \mathrm{I} ; \mathrm{N}]$} & {$[600 ; 47 ; 29 ; 46 ; 52 ; 22]$} \\
\hline $\mathrm{R}-13$ & 0.25 & 7 & {$[\mathrm{I}]$} & {$[109]$} \\
\hline $\mathrm{R}-17$ & 0.25 & 7 & {$[\mathrm{I}]$} & {$[69]$} \\
\hline R-19 & 0.25 & 7 & {$[\mathrm{~K} ; \mathrm{J}]$} & {$[435 ; 35]$} \\
\hline R-20 & 0.25 & 7 & {$[\mathrm{M}]$} & {$[17]$} \\
\hline $\mathrm{R}-21$ & 0.25 & 7 & {$[\mathrm{~L}]$} & {$[22]$} \\
\hline $\mathrm{R}-23$ & 60 & 7 & {$[\mathrm{I}]$} & {$[54]$} \\
\hline $\mathrm{R}-24$ & 60 & 7 & {$[\mathrm{~J} ; \mathrm{K}]$} & {$[12 ; 227]$} \\
\hline $\mathrm{R}-26$ & 0.25 & 7 & {$[\mathrm{I} ; \mathrm{F} ; \mathrm{K}]$} & {$[242 ; 39 ; 251]$} \\
\hline $\mathrm{R}-34$ & 0.25 & 7 & {$[\mathrm{H}]$} & {$[80]$} \\
\hline $\mathrm{R}-37$ & 0.25 & 7 & {$[\mathrm{~A}]$} & {$[306]$} \\
\hline $\mathrm{R}-40$ & 0.25 & 7 & {$[\mathrm{~J}]$} & {$[92]$} \\
\hline $\mathrm{R}-46$ & 0.25 & 7 & {$[\mathrm{H}]$} & {$[72]$} \\
\hline R-52 & 0.25 & 7 & {$[\mathrm{~L}]$} & [292] \\
\hline $\mathrm{R}-53$ & 0.25 & 7 & {$[\mathrm{M}]$} & {$[25]$} \\
\hline $\mathrm{R}-57$ & 0.25 & 7 & {$[\mathrm{~J} ; \mathrm{K}]$} & {$[36 ; 140]$} \\
\hline
\end{tabular}

$C_{\text {seq }}$ was corrected using the error correction framework. The error in energy estimation $(e)$ was found to fluctuate between \pm 70 for all appliances once error correction was applied. Without error correction, as showcased by Table 3 , the error values $e^{\prime}$ can fluctuate drastically. On average, using the framework improved the energy estimates for 11 houses, did not have any impact on 6 houses, and impacted 2 houses negatively. The average negative impact $e-e^{\prime}$ was $-8.2 \%$, which is a trivial trade-off compared to the positive improvements 


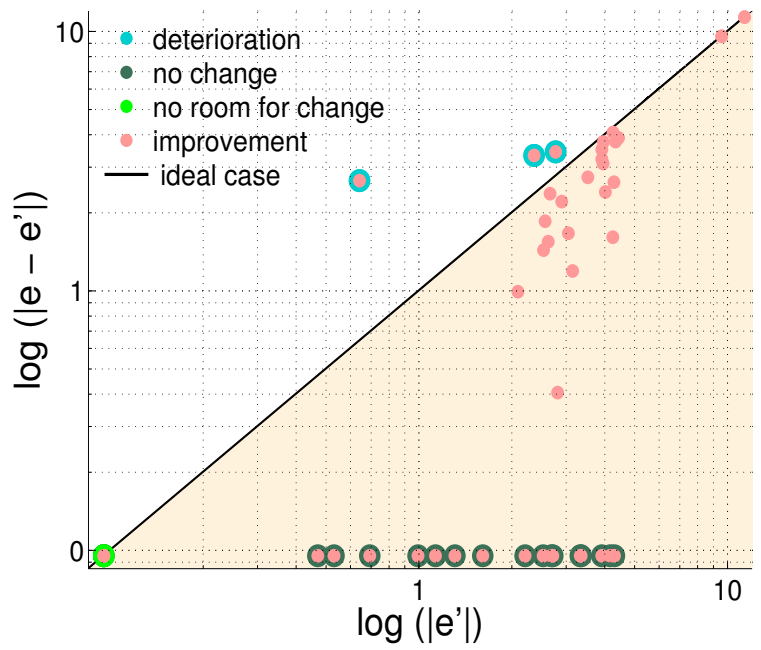

Figure 10: A log-log plot of the change in energy estimation error upon using the framework (y-axis) and the energy estimation error without the framework (x-axis). Appliance \# denotes the S.N. as shown in Table 3

in energy estimation. Figure 10 illustrates these results in a log-log plot of the energy estimation error without error correction $\left(e^{\prime}\right)$ and the change in energy estimation because of the error correction framework $\left(\left|e-e^{\prime}\right|\right)$. The line $(y=x)$ denotes the ideal case, where all the errors in energy estimation process is accounted for by the error correction step. The shaded area: $x>y$ denotes regions where use of the error correction framework positively impacts the energy estimation process, by accounting for a portion of the errors. The zero values denote cases where there was no change in energy estimation error upon using the error correction framework. Points in the region: $x<y$ show cases where using error correction adversely affects the energy estimation results. Again, it can be seen that, on average, the framework has a positive effect on energy estimation for appliances.

\section{Discussion}

The analysis on simulated and real data show that there is significant improvement in the process of energy estimation upon using an error correction framework. Figure 8, for instance, shows that the DTW distance of corrected sequence is consistently lower than that of corrupted sequence over varying number of states. This was found to be true for other parameters in the simulation, including sequence length, and probability threshold. Similarly, and perhaps, as expected, the same was found of energy estimation results and RMS distance. As can be seen in Figure 10, there are certain instances where using the framework negatively affects the energy estimation. Typically, these are instances where the event labels (and, consequently, sequence $C_{\text {seq }}^{E}$ ) were almost perfect, which is not reflective of a real world scenario. In addition, in some of these 
Table 3: Results of energy estimation on 43 appliances from 19 houses with and without the error correction framework. The sequence for error correction, and subsequent energy estimation was done using the framework discussed in 13. Dataset index B stands for BLUED and R stands for REDD. S.N is the appliance \# as depicted in Figure 10 Definition of $\mathbf{e}, \mathbf{e}^{\prime}$ and $\Delta \mathbf{e}$ can be found in Equations 7,8 and 9 respectively.

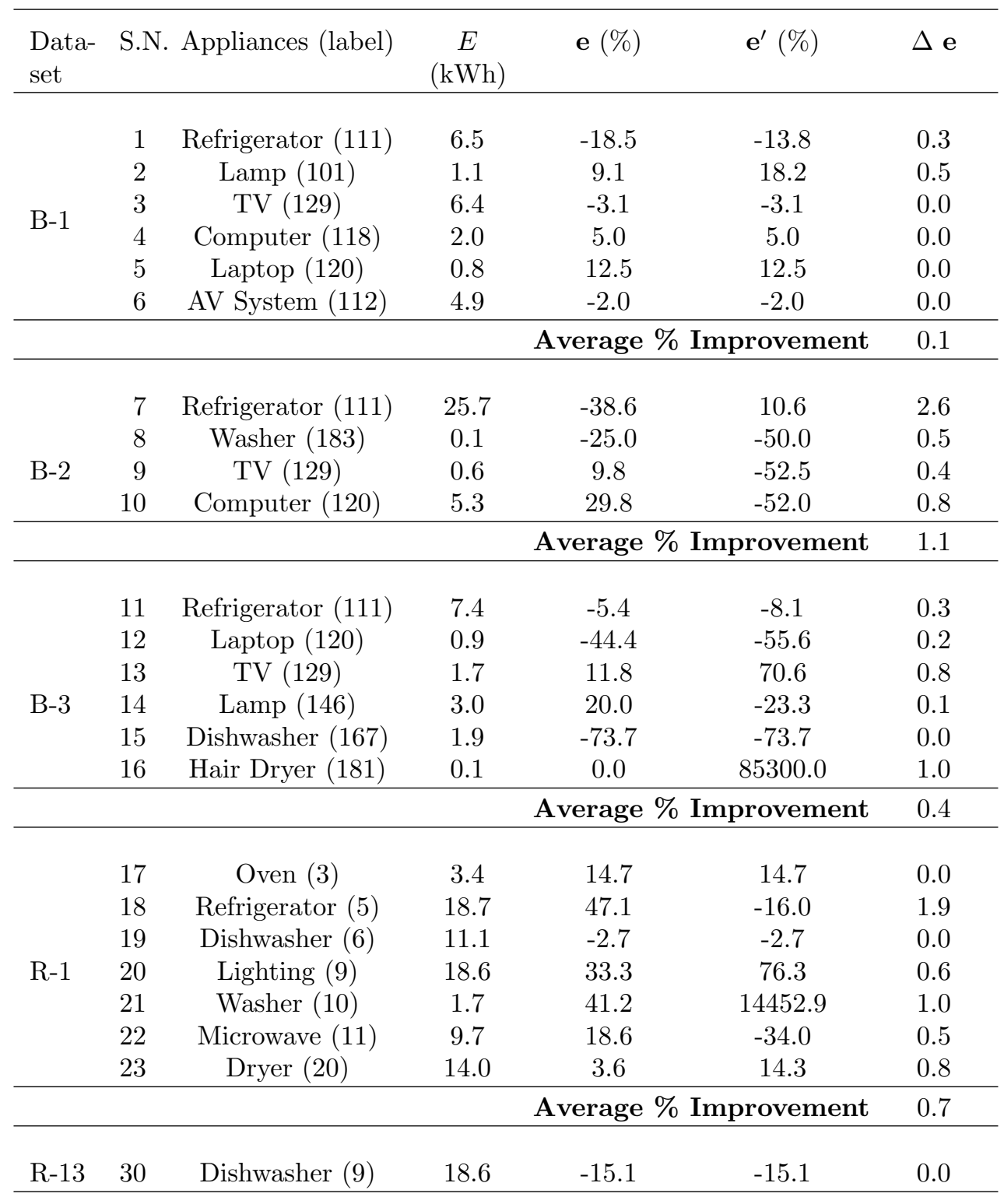




\begin{tabular}{|c|c|c|c|c|c|c|}
\hline $\begin{array}{l}\text { Data- } \\
\text { set }\end{array}$ & S.N & $\begin{array}{c}\text { Appliances } \\
\text { (label) }\end{array}$ & $\begin{array}{c}E \\
(\mathrm{kWh})\end{array}$ & e $(\%)$ & $\mathbf{e}^{\prime}(\%)$ & $\Delta \mathbf{e}$ \\
\hline $\mathrm{R}-17$ & 31 & Dishwasher (11) & 6.6 & -18.2 & -16.7 & 0.1 \\
\hline R-19 & $\begin{array}{l}32 \\
33\end{array}$ & $\begin{array}{c}\text { Dryer }(12) \\
\text { Microwave (15) }\end{array}$ & $\begin{array}{c}34.5 \\
0.1\end{array}$ & $\begin{array}{c}-6.7 \\
-64.5\end{array}$ & $\begin{array}{l}-13.0 \\
-64.5\end{array}$ & $\begin{array}{l}0.5 \\
0.0\end{array}$ \\
\hline & & & & \multicolumn{2}{|c|}{ Average \% Improvement } & 0.3 \\
\hline $\mathrm{R}-20$ & 34 & Pool Pump (0) & 42.0 & 16.2 & -1.9 & 7.5 \\
\hline $\mathrm{R}-21$ & 35 & Furnace $(0)$ & 2.9 & 58.6 & 72.4 & 0.2 \\
\hline $\mathrm{R}-23$ & 24 & Dishwasher (0) & 1.9 & -15.8 & -21.1 & 0.3 \\
\hline \multirow[t]{2}{*}{$\mathrm{R}-24$} & $\begin{array}{l}25 \\
26\end{array}$ & $\begin{array}{c}\text { Microwave }(1) \\
\text { Dryer }(12)\end{array}$ & $\begin{array}{c}1.2 \\
14.3\end{array}$ & $\begin{array}{c}0.0 \\
-16.8\end{array}$ & $\begin{array}{c}0.0 \\
-12.6\end{array}$ & $\begin{array}{l}0.0 \\
0.3\end{array}$ \\
\hline & & & & \multicolumn{2}{|c|}{ Average \% Improvement } & 0.2 \\
\hline \multirow[t]{2}{*}{$\mathrm{R}-26$} & $\begin{array}{l}27 \\
28 \\
29\end{array}$ & $\begin{array}{c}\text { Dishwasher (1) } \\
\text { AV Closet (17) } \\
\text { Dryer (18) }\end{array}$ & $\begin{array}{l}1.1 \\
8.8 \\
8.2\end{array}$ & $\begin{array}{c}-9.1 \\
-35.2 \\
-28.0\end{array}$ & $\begin{array}{c}-9.1 \\
-84.1 \\
-28.0\end{array}$ & $\begin{array}{l}0.0 \\
0.6 \\
0.0\end{array}$ \\
\hline & & & & \multicolumn{2}{|c|}{ Average \% Improvement } & 0.2 \\
\hline $\mathrm{R}-34$ & 36 & Oven(17) & 2.7 & 3.7 & 3.7 & 0.0 \\
\hline $\mathrm{R}-37$ & 37 & Fridge(3) & 0.6 & -16.7 & -50.0 & 0.7 \\
\hline $\mathrm{R}-40$ & 38 & Microwave (4) & 2.0 & -50.0 & -50.0 & 0.0 \\
\hline R-46 & 39 & Oven (4) & 2.7 & 0.0 & 0.0 & 0.0 \\
\hline $\mathrm{R}-52$ & 40 & Furnace (2) & 2.0 & -65.0 & -70.0 & 0.1 \\
\hline $\mathrm{R}-53$ & 41 & Pool Pump (0) & 31.9 & 1.6 & 1.6 & 0.0 \\
\hline $\mathrm{R}-57$ & $\begin{array}{l}42 \\
43\end{array}$ & $\begin{array}{c}\text { Microwave }(1) \\
\text { Dryer }(12)\end{array}$ & $\begin{array}{c}0.7 \\
17.6\end{array}$ & $\begin{array}{c}-28.6 \\
1.7\end{array}$ & $\begin{array}{c}-28.6 \\
1.7\end{array}$ & $\begin{array}{l}0.0 \\
0.0\end{array}$ \\
\hline & & & & Average & provement & 0.0 \\
\hline
\end{tabular}


cases, the errors that are present are valid under the FSM model, and are hence not detected as errors by the algorithm. Overall, however, the positive gain in energy estimation instances (mean: $5266.8 \%$, median $15.5 \%$ ), far outweighs the negative impacts (mean: $13.9 \%$, median: $9.5 \%$ ). For 17 appliances, using the framework doesn't change the energy estimation results. This is mostly because the labels are accurate enough to begin with and error correction is not required.

The major parameters that affect the energy estimation results are the cost constants for substitution, insertion and deletion. It was found that lower values of insertion and deletion costs mean that the variance is low in energy estimation errors as shown in Figure 7. The median error was low for most combinations of these two costs. The only information the error correction framework has about the noise in the channel is about state transitions that are not possible. Due to this inherent difficulty of the problem, sometimes, there are erroneous sequences that satisfy the FSM under which the sequence is operating, and hence don't show up as errors. As a result, there is potential for errors to be accumulated and the energy estimates to be significantly off from ground truth. Hence, we used median of 100 trials as the representative of each cost combination, as means are swayed heavily by outliers. Figuring out sequences (or sequence segments) that are highly unlikely but still probable under operating FSM model of the appliance, and treating those are errors is an interesting extension to the problem. One way to achieve this is to create a likelihood metric for an observed sequence, and assume it to be erroneous if it lies under a certain threshold. Then, based on certain beliefs, assign specific points in the sequence as error points, and mold the problem into the framework described above. We leave such implementations as future work.

Another underlying but understated assumption that has been prevalent throughout the framework is that the sequences are long enough to reflect all possible appliance states and transitions enough times for a model to be created. Typically, this means at least a few days worth of data has to be collected from the appliance for energy to be estimated. Given the main goal of NILM is to give disaggregated feedback at the end of certain set time-frames (typically ranging from week to month), assuming the availability of such data is not impractical. But there can be scenarios where a real time model generation with the available data and subsequent energy estimation might be required. Our models have not been tested for such implementations, and we leave such extension as future work as well. The model has also implicitly assumed that the initial and the final nodes of the sequence $\left(c_{1}\right.$ and $\left.c_{m}\right)$ are fixed not erroneous. Obviously, this is not guaranteed. A more robust way to find the starting and ending nodes for correction might be to utilize the aggregate power information, and track it for baselines (also known as background power). Once a reasonable baseline has been established, every time the aggregate power consumption reaches the baseline, it is guaranteed that the last event from the appliance is an off from its previous state, and the next event will be from off to a state of positive power consumption. Same information about baselines can also be utilized to 
constrain the energy estimates in the shortest path algorithm. Since finding appropriate baselines automatically in aggregate power consumption data is a complex problem in its own right, and an active field of research, we have steered away from utilizing that such information in our models at this point.

The way in which we have incorporated the FSM model into the error correction process was by iteratively and locally modifying the costs at each instance where the FSM states were violated. Although this method worked fairly well, a more efficient method is to modify the shortest path search in such a way that this check is performed at each node, and hence nodes that violate the maximum and minimum states level are not selected in the shortest path in the first iteration itself. The trade-offs of complexity of such an approach versus potential gains is an interesting study in itself, and we hope to explore it in the future.

One of the issues that has hindered comparison of different proposed NILM solutions and frameworks is the lack of standard datasets. In this paper, we have used publicly available datasets where possible. Additionally, we have extended the repository of publicly available datasets for supervised NILM by labeling events manually when labels were not available. We will be making all the datasets, labels, and code utilized in this paper publicly available as well. Energy estimation is an integral part of supervised NILM, and clearly a robust error correction framework can improve the results of the whole disaggregation process. Although our focus has been mostly on energy estimation, this method of using domain specific beliefs to correct for errors in sequences generated by processes whose models are known could be extended to other fields like DNA sequencing, etc.

\section{Conclusion}

In this paper, we presented a framework for correcting errors in sequences generated for energy estimation in supervised NILM. Although we focus our analysis on the sequence generated by one particular framework, the method is agnostic to how the sequences are generated. The method utilizes prior beliefs about how appliances behave in general and incorporates that to a network flow to minimize the cost of correcting errors in the sequence. We tested our framework on simulated data to study how different cost parameters affect the performance and found that over a wide range of values, using the framework yields better results. We further tested the framework on real data from 43 different appliances collected from 19 houses, and found that energy estimation results improve on average upon using the error correction framework. In addition to energy estimation in supervised NILM, this error correction framework can be utilized in energy auditing using external sensors, validating appliance labels generated by other processes, etc. 


\section{Acknowledgments}

Mario Bergés was partially supported by the National Science Foundation (NSF) Grant \#09-30868 while working on the results presented in this manuscript. The opinions presented here are those of the authors and not of the National Science Foundation.

\section{References}

[1] M. Zeifman, K. Roth, Nonintrusive appliance load monitoring: Review and outlook, in: 2011 IEEE International Conference on Consumer Electronics (ICCE), 2011, pp. 239 -240. doi:10.1109/ICCE.2011.5722560.

[2] A. Zoha, A. Gluhak, M. Imran, S. Rajasegarar, Non-intrusive load monitoring approaches for disaggregated energy sensing: A survey, Sensors 12 (12) (2012) 16838-16866. doi:10.3390/s121216838 URL http://www .mdpi .com/1424-8220/12/12/16838

[3] M. J. Johnson, A. S. Willsky, Bayesian nonparametric hidden semi-markov models, The Journal of Machine Learning Research 14 (1) (2013) 673-701.

[4] J. Z. Kolter, T. Jaakkola, Approximate inference in additive factorial hmms with application to energy disaggregation, in: International conference on artificial intelligence and statistics, 2012, pp. 1472-1482.

[5] H. Lange, M. Berges, Efficient Inference in Dual-emission FHMM for Energy Disaggregation, in: Workshops at the Thirtieth AAAI Conference on Artificial Intelligence, Phoenix, Arizona, USA, 2016.

[6] European non-intrusive load monitoring workshop, http://nilm.eu/, accessed: 2016-10-01.

[7] International workshop on non-intrusive load monitoring, http:// nilmworkshop.org/, accessed: 2016-10-01.

[8] G. Hart, Nonintrusive appliance load monitoring, Proceedings of the IEEE 80 (12) (1992) 1870-1891.

[9] S. Gupta, M. S. Reynolds, S. N. Patel, Electrisense: single-point sensing using emi for electrical event detection and classification in the home, in: Proceedings of the 12th ACM international conference on Ubiquitous computing, ACM, 2010, pp. 139-148.

[10] S. B. Leeb, J. L. Kirtley Jr, Transient event detector for use in non-intrusive load monitoring systems, uS Patent 5,483,153 (Jan. 9 1996).

[11] M. E. Berges, E. Goldman, H. S. Matthews, L. Soibelman, Enhancing electricity audits in residential buildings with non-intrusive load monitoring, Journal of industrial ecology 14 (5) (2010) 844-858. 
[12] F. Jazizadeh, B. Becerik-Gerber, M. Berges, L. Soibelman, An unsupervised hierarchical clustering based heuristic algorithm for facilitated training of electricity consumption disaggregation systems, Advanced Engineering Informatics 28 (4) (2014) 311-326.

[13] S. Giri, M. Berges, An energy estimation framework for eventbased methods in non-intrusive load monitoring, Energy Conversion and Management 90 (0) (2015) 488 - 498. doi:http: $/ /$ dx.doi.org/10.1016/j.enconman.2014.11.047.

URL http://www.sciencedirect.com/science/article/pii/ S0196890414010164

[14] K. Anderson, A. Ocneanu, D. Benitez, D. Carlson, A. Rowe, M. Berges, Blued: A fully labeled public dataset for \{Event-Based $\{$ Non-Intrusive load monitoring research, 2012.

[15] F. J. MacWilliams, N. J. A. Sloane, The Theory of Error Correcting Codes, Elsevier, 1977.

[16] X. Yang, S. P. Chockalingam, S. Aluru, A survey of error-correction methods for next-generation sequencing, Briefings in Bioinformatics 14 (1) (2013) 56-66. doi:10.1093/bib/bbs015.

[17] G. W. Hart, Minimum information estimation of structure, Thesis, Massachusetts Institute of Technology, thesis (Ph. D.)-Massachusetts Institute of Technology, Dept. of Electrical Engineering and Computer Science, 1987. (1987). URL http://dspace.mit.edu/handle/1721.1/14792

[18] K. He, L. Stankovic, J. Liao, V. Stankovic, Non-intrusive load disaggregation using graph signal processing, IEEE Transactions on Smart Grid PP (99) (2016) 1-1. doi:10.1109/TSG.2016.2598872.

[19] A. Sandryhaila, J. M. F. Moura, Discrete signal processing on graphs, IEEE Transactions on Signal Processing 61 (7) (2013) 1644-1656. doi:10.1109/ TSP.2013.2238935,

[20] A. Sandryhaila, J. M. F. Moura, Big data analysis with signal processing on graphs: Representation and processing of massive data sets with irregular structure, IEEE Signal Processing Magazine 31 (5) (2014) 80-90. doi: 10.1109/MSP. 2014.2329213

[21] S. Chen, R. Varma, A. Sandryhaila, J. Kovacevic, Discrete signal processing on graphs: Sampling theory, IEEE Transactions on Signal Processing 63 (24) (2015) 6510-6523. doi:10.1109/TSP.2015.2469645

[22] G. Hart, A. Bouloutas, Correcting dependent errors in sequences generated by finite-state processes, IEEE Transactions on Information Theory 39 (4) (1993) 1249-1260. doi:10.1109/18.243442. 
[23] M. S. Bazaraa, J. J. Jarvis, H. D. Sherali, Linear Programming and Network Flows, John Wiley \& Sons, 2011.

[24] E. W. Dijkstra, A note on two problems in connexion with graphs, Numerische Mathematik 1 (1) (1959) 269-271. doi:10.1007/BF01386390. URL http://link. springer.com/article/10.1007/BF01386390

[25] R. K. Ahuja, K. Mehlhorn, J. B. Orlin, R. E. Tarjan, Faster algorithms for the shortest path problem, Journal of the Association for Computing Machinery 37 (2) (1990) 213-223.

URL http://cat.inist.fr/?aModele=afficheN\&cpsidt=19245968

[26] M. L. Fredman, R. E. Tarjan, Fibonacci heaps and their uses in improved network optimization algorithms, J. ACM 34 (3) (1987) 596-615. doi: 10.1145/28869.28874.

URL http://doi.acm.org/10.1145/28869.28874

[27] H. Sakoe, S. Chiba, Dynamic programming algorithm optimization for spoken word recognition, IEEE Transactions on Acoustics, Speech and Signal Processing 26 (1) (1978) 43-49. doi:10.1109/TASSP.1978.1163055.

[28] D. J. Burr, Designing a handwriting reader, IEEE Trans. Pattern Anal. Mach. Intell. 5 (5) (1983) 554-559. doi:10.1109/TPAMI.1983.4767435 URL http://dx.doi.org/10.1109/TPAMI .1983.4767435

[29] J. Liao, G. Elafoudi, L. Stankovic, V. Stankovic, Non-intrusive appliance load monitoring using low-resolution smart meter data, in: 2014 IEEE International Conference on Smart Grid Communications (SmartGridComm), 2014, pp. 535-540. doi:10.1109/SmartGridComm.2014.7007702.

[30] F. Jazizadeh, B. Becerik-Gerber, M. Berges, L. Soibelman, An unsupervised hierarchical clustering based heuristic algorithm for facilitated training of electricity consumption disaggregation systems, Advanced Engineering Informatics 28 (4) (2014) 311-326. doi:10.1016/j . aei.2014.09.004.

URL http://www.sciencedirect.com/science/article/pii/ S1474034614000913

[31] Z. Kolter, M. Johnson, Redd: A public data set for energy disaggregation research, in: In proceedings of the SustKDD workshop on Data Mining Applications in Sustainability, 2011. 